\title{
VI Jornada de Cuidados Paliativos do INCA
}

VI INCA Conference on Palliative Care

VI Jornada de Cuidados Paliativos del INCA

Data: 25 de outubro de 2016.

Local: Hospital do Câncer III/Instituto Nacional de Câncer José Alencar Gomes da Silva (INCA)/Ministério da Saúde (MS). Auditório Gama Filho. Rua Visconde de Santa Isabel, n² 274 - Vila Izabel. Rio de Janeiro (RJ), Brasil. 


\title{
Fatores Associados à Sobrevida de Pacientes com Câncer Avançado em Cuidados Paliativos
}

Factors Associated with Survival of Advanced Cancer Patients in Palliative Care

Factores Relacionados a la Supervivencia de los Pacientes con Cáncer Avanzado

en los Cuidados Paliativos

\author{
Emanuelly Varea Maria Wiegert ${ }^{1}$ L Livia Costa de Oliveira ${ }^{2}$; Larissa Calixto-Lima ${ }^{3}$; Natália Alvarenga Borges ${ }^{4}$
}

\begin{abstract}
Resumo
Introdução: Em cuidados paliativos, é fundamental identificar fatores clínicos que possam auxiliar na predição de sobrevida. Objetivo: Avaliar fatores associados à sobrevida de pacientes oncológicos em cuidados paliativos. Método: Estudo observacional, com pacientes a partir de 20 anos, avaliados na Unidade de Cuidados Paliativos do Instituto Nacional de Câncer José Alencar Gomes da Silva (INCA) entre junho e agosto de 2016. A sobrevida foi determinada pelo tempo em dias contados da data da avaliação até o óbito/censura. Foi empregada análise de regressão linear bivariada tendo como variáveis independentes medidas de estado nutricional, funcionalidade e marcadores inflamatórios. As análises estatísticas foram realizadas no programa STATA 12.0. Estudo aprovado pelo Comitê de Ética em Pesquisa do INCA. Resultados: Foram estudados 114 pacientes, média de idade $60,8( \pm 1,2)$ anos, 59,6\% feminino, mediana de sobrevida 34 (2-154) dias. Os fatores que associaram-se positivamente com a sobrevida foram: adequação da dobra cutânea tricipital ( $\beta=3,92 ; \mathrm{IC} 95 \%: 0,98 ; 6,84)$ e da circunferência muscular do braço $(\beta=5,64 ; \mathrm{IC} 95 \%: 0,11 ; 11,17)$, Karnofsky Performance Status ( $\beta=0,80$; IC95\%:0,51;1,09) e albumina ( $\beta=12,84 ;$ IC95\%:7,39;18,29). Outrossim, o escore total da Avaliação Subjetiva Global Produzida pelo Próprio Paciente $(\beta=-1,19 ; \mathrm{IC} 95 \%:-1,93 ;-0,46)$ e seus domínios relativos à ingestão alimentar $(\beta=-3,91 ; \mathrm{IC} 95 \%$ :-7,47;-0,34), sintomas $(\beta=-1,66$; IC95\%:-2,73; -0,59) e capacidade funcional ( $\beta=-8,85 ;$ IC95\%:-13,62;-4,07), assim como o Escore Prognóstico de Glasgow $(\beta=-7,89 ;$ IC95\%:-13,36;-2,42) e a proteína C-Reativa ( $\beta=-0,68$; IC95\%:-1,20;-0,17) associaram-se negativamente. Conclusáo: $O$ pior estado nutricional, a capacidade funcional reduzida e a gravidade da inflamação sistêmica associaram-se à menor sobrevida nessa população. Palavras-chave: Análise de Sobrevida; Cuidado Paliativo; Neoplasias/terapia; Estado Nutricional; Prognóstico.
\end{abstract}

\section{Declaração de Conflito de Interesses: Nada a Declarar.}

\footnotetext{
${ }^{1}$ Nutricionista. Mestre em Nutrição Humana pela Universidade Federal do Rio de Janeiro (UFRJ). Serviço de Nutrição e Dietética do Hospital do Câncer IV (HC IV)/Instituto Nacional de Câncer José Alencar Gomes da Silva (INCA)/Ministério da Saúde (MS). Rio de janeiro (RJ), Brasil. E-mail: ewiegert@inca.gov.br

${ }^{2}$ Nutricionista. Doutora em Ciência Nutricionais pela UFRJ. Serviço de Nutrição e Dietética do HC IV/INCA/MS. Rio de janeiro (RJ), Brasil. E-mail: livia.oliveira@inca.gov.br

${ }^{3}$ Nutricionista. Mestre em Nutrição pela Universidade do Estado do Rio de Janeiro (UERJ). Serviço de Nutrição e Dietética do HC IV/INCA/MS. Rio de janeiro (RJ), Brasil.E-mail: larissa.lima@inca.gov.br

${ }^{4}$ Nutricionista. Doutora em Ciências Médicas pela Universidade Federal Fluminense (UFF). Rio de janeiro (RJ), Brasil. E-mail: nat_borges_@hotmail.com Endereço para correspondência: Emanuelly Varea Maria Wiegert. Rua Visconde de Santa Isabel, no 274 - Vila Isabel. Rio de Janeiro (RJ), Brasil. CEP:

20560-120.E-mail: ewiegert@inca.gov.br
} 


\title{
Relação do Estado Nutricional e da Resposta Inflamatória Sistêmica com a Sobrevida de Pacientes com Câncer Avançado em Cuidados Paliativos
}

\author{
Relationship of Nutritional Status and Systemic Inflammatory Response with \\ Survival of Advanced Cancer Patients in Palliative Care \\ La Relación entre el Estado Nutricional y la Respuesta Inflamatoria Sistémica con \\ la Supervivencia de los Pacientes con Cáncer Avanzado en los Cuidados Paliativos
}

\author{
Emanuelly Varea Maria Wiegert ${ }^{1}$; Livia Costa de Oliveira ${ }^{2}$; Larissa Calixto-Lima ${ }^{3}$; Mariana dos Santos Campello Queiroz ${ }^{4}$
}

\begin{abstract}
Resumo
Introduçáo: A avaliação prognóstica é indispensável na prática clínica dos cuidados paliativos. Objetivo: Avaliar a relaçáo do estado nutricional e da resposta inflamatória sistêmica com a sobrevida de pacientes oncológicos em cuidados paliativos. Método: Estudo observacional, com pacientes a partir de 20 anos, avaliados na Unidade de Cuidados Paliativos do Instituto Nacional de Câncer José Alencar Gomes da Silva (INCA) entre junho e agosto de 2016. A resposta inflamatória sistêmica foi determinada por meio do escore prognóstico de Glasgow (EPG) modificado, cuja classificação varia de 0 a 2 conforme relação entre valores séricos de Proteína C-Reativa (PCR) e albumina; e o estado nutricional pela Avaliação Subjetiva Global Produzida Pelo Paciente (ASG-PPP). A sobrevida foi determinada pelo tempo em dias contados da data da avaliação até o óbito/censura. Foram realizadas análises de regressão logística bivariada e multivariada de Cox utilizando o programa Stata 12.0. Estudo aprovado pelo Comitê de Ética em Pesquisa do INCA. Resultados: Foram avaliados 114 pacientes, média de idade de $60,8( \pm 1,3)$ anos, dos quais $38,3 \%$ possuíam EPG $\geq 1$ e $82,3 \%$ eram desnutridos (ASG-PPP B/C). Observou-se que EPG $\geq 1$ (OR:5,04; IC 95\%:2,14-11,88), PCR > 10 (OR:6,67; IC 95\%:2,81-15,80) e ASG-PPP B/C (OR:3,38; IC 95\%:1,79-6,36) associaram-se ao óbito em 30 dias. Ademais, EPG $\geq 1$ (HR:0,14; IC 95\%:0,03-0,63), ASG-PPP B/C (HR:2,68; IC 95\%:1,50-4,79) e PCR > 10 (HR:21,90; IC 95\%:4,58-104,66) demonstraram-se fatores prognósticos independentes para a sobrevida. Conclusão: $O$ pior estado nutricional e a gravidade da inflamaçáo sistêmica constituem-se fatores prognósticos úteis na populaçáo estudada.
\end{abstract}

Palavras-chave: Análise de sobrevida, Neoplasias/terapia; Estado Nutricional; Inflamação; Cuidados paliativos.

\section{Declaraçáo de Conflito de Interesses: Nada a Declarar.}

\footnotetext{
${ }^{1}$ Nutricionista. Mestre em Nutrição Humana pela Universidade Federal do Rio de Janeiro (UFRJ). Serviço de Nutriçấo e Dietética do Hospital do Câncer IV (HC IV)/Instituto Nacional de Câncer José Alencar Gomes da Silva (INCA)/Ministério da Saúde (MS). Rio de janeiro (RJ), Brasil. E-mail: ewiegert@inca.gov.br

${ }^{2}$ Nutricionista. Doutora em Ciência Nutricionais pela UFRJ. Serviço de Nutrição e Dietética do HC IV/INCA/MS. Rio de janeiro (RJ), Brasil. E-mail: livia.oliveira@inca.gov.br

${ }^{3}$ Nutricionista. Mestre em Nutriçấo pela Universidade do Estado do Rio de Janeiro (UERJ). Serviço de Nutriçấo e Dietética do HC IV/INCA/MS. Rio de janeiro (RJ), Brasil. E-mail: larissa.lima@inca.gov.br

${ }^{4}$ Nutricionista pela Universidade Veiga de Almeida (UVA). Rio de janeiro (RJ), Brasil. E-mail: marianacampello561@yahoo.com.br

Enderȩ̧o para correspondência: Emanuelly Varea Maria Wiegert. Rua Visconde de Santa Isabel, no 274 - Vila Isabel. Rio de Janeiro (RJ), Brasil. CEP:

20560-120.E-mail: ewiegert@inca.gov.br
} 


\title{
Cuidados Paliativos em Fim de Vida: Reflexões sobre a Prática do Assistente Social \\ End-of-Life Palliative Care: Reflections on the Practice of Social Worker \\ Cuidados Paliativos al Final de la Vida: Reflexiones sobre la Práctica del \\ Trabajador Social
}

Andrea Frossard '; Alessandra Gomes ${ }^{2}$ : Luciana Rodrigues ${ }^{3}$; Marli Schaefer ${ }^{4}$; Erika Palmieri ${ }^{5}$

\begin{abstract}
Resumo
Introdução: Estudo desenvolvido no Núcleo de Estudos Integrados em Cuidados Paliativos do Hospital do Câncer IV do Instituto Nacional de Câncer José Alencar Gomes da Silva (HC IV/INCA). Considerando a dimensão técnico-operativa, reflete-se sobre a ação profissional vinculada aos cuidados paliativos. Objetivos: Compreender os cuidados paliativos em fim de vida como integrante dos cuidados paliativos e identificar os processos interventivos do Serviço Social nesse âmbito. Métodos: Estudo qualitativo, utilizando-se da análise temática de conteúdo (à luz da hermenêutica-dialética) na perspectiva teórico-crítica de veio marxiano. Resultados: A partir do desnudamento dos núcleos temáticos (NT) significativos vinculados à dimensão técnico-operativa (NT1 - situação de indigência e estado de pobreza e a intervenção profissional; NT2 - família, novos arranjos e a relação afetividade e afinidade na gestáo do cuidado; NT3 - diferentes modalidades de atendimento assistenciais e os cuidados em fim de vida; e NT4 especificidades dos instrumentais técnico-operativos utilizados no cotidiano profissional), foi possível o desenvolvimento da investigação cujo conteúdo deriva-se do NT3. Conclusáo: É necessário o incremento da produção acadêmica no Serviço Social no país, à luz das requisiçôes por parte de pacientes e suas famílias, da equipe de saúde e do processo de integração da rede de cuidados em cuidados paliativos desde a elaboração de protocolos (como, por exemplo, óbito de população em estado de pobreza ou de indigência) até o desenvolvimento de pesquisas estratégicas em consonância com a realidade nacional.
\end{abstract}

Palavras-chave: Cuidados Paliativos; Cuidados Paliativos na Terminalidade da Vida; Serviço Social/métodos; Doente Terminal.

\section{Declaraçáo de Conflito de Interesses: Nada a Declarar.}

\footnotetext{
${ }^{1}$ Assistente Social do Hospital do Câncer IV (HC IV)/ Instituto Nacional de Câncer José Alencar Gomes da Silva (INCA)/Ministério da Saúde (MS). Rio de Janeiro (RJ), Brasil. Pós-Doutora em Ciências Humanas Universidade Federal de Santa Catarina (UFSC). Florianópolis (SC), Brasil. E-mail: afrossard@inca.gov.br

${ }^{2}$ Assistente Social do HC IV/INCA/MS. Especialista pela Universidade Cândido Mendes (UCAM). Rio de Janeiro (RJ), Brasil. E-mail: alessandra.carvalho@inca.gov.br ${ }^{3}$ Assistente Social do HC IV/INCA/MS. Residente do INCA/MS. Rio de Janeiro (RJ), Brasil. E-mail: Luciana_seso@hotmail.com

${ }^{4}$ Assistente Social do HC IV/INCA/MS. Rio de Janeiro (RJ), Brasil. Especialista pela Universidade de Brasília (UnB). Brasília (DF), Brasil. E-mail: marlischaeffer22@gmail.com

${ }_{5}^{5}$ Assistente Social do HC IV/INCA/MS. Rio de Janeiro (RJ), Brasil. Especialista pela Universidade Federal de Pernambuco (UFPE). Recife (PE), Brasil. Rio de Janeiro (RJ), Brasil. E-mail: erika_fontes@hotmail.com

Enderȩ̧o para correspondência: Andrea Frossard. Rua Visconde de Santa Isabel, no 274 - Vila Isabel. Rio de Janeiro (RJ), Brasil. CEP: 20560-120. E-mail:

afrossard@inca.gov.br
} 


\title{
Espiritualidade e Cuidados Paliativos: uma Proposta de Humanização do Cuidado Spirituality and Palliative Care: a Humanization Proposal of Care
} Espiritualidad y Cuidados Paliativos: una Propuesta para la Humanización del Cuidado

\author{
Renata Carla Nencetti Pereira Rocha ${ }^{1}$; Eliane Ramos Pereiraª ${ }^{2}$ Rose Mary Costa Rosa Andrade Silva ${ }^{3}$; Luciana Aparecida Faria de Oliveira ${ }^{4}$
}

\begin{abstract}
Resumo
Introduçáo: A presença de um indivíduo com câncer em cuidados paliativos oncológicos provoca no seio familiar uma série de vicissitudes e sofrimentos relacionados à proximidade da morte. Nesse prisma, é sobre o cuidador (comumente, um familiar) que recaem as exigências práticas e emocionais da doença. Desse modo, a espiritualidade tem sido acionada como instrumento nas situaçôes complexas e existenciais das famílias e sua abordagem torna-se cada vez mais requisitada à prática assistencial à saúde. Objetivo: Conhecer a influência da espiritualidade na vida dos cuidadores familiares de pacientes em cuidados paliativos oncológicos. Método: Revisão integrativa da literatura, realizada por meio de busca nas bases de dados Literatura Latino-Americana e do Caribe em Ciências da Saúde e Medical Literature Analysis and Retrieval System Online, sendo encontradas 37 publicaçôes, das quais dez corresponderam esta revisão. Resultado: Cuidadores buscam, por meio da espiritualidade, consolo, força e sentido para as suas vidas e é ela quem os direcionará nessa dimensão e contexto. Como recurso de enfrentamento, os mesmos utilizam a força da fé, crenças e práticas religiosas/espirituais. Em relação à assistência espiritual desejada pelo enfermeiro, os cuidadores relataram a escuta profunda, empatia, respeito, humor e momento de silêncio. Conclusáo: Evidenciou-se que, em geral, os cuidadores se apropriam da espiritualidade como recurso para enfrentamento do câncer. Contudo, os cuidadores familiares são os que mais requisitam os serviços de capelania no campo dos cuidados paliativos. Registra-se, ainda, que há necessidade de produçóes científicas que abordem o apoio espiritual do enfermeiro em prol da humanização do cuidado.
\end{abstract}

Palavras-chave: Espiritualidade; Cuidados Paliativos; Cuidadores; Neoplasias.

\section{Declaraçáo de Conflito de Interesses: Nada a Declarar.}

\footnotetext{
${ }^{1}$ Enfermeira-Paliativista do Hospital do Câncer IV (HC IV)/Instituto Nacional de Câncer José Alencar Gomes da Silva (INCA)/Ministério da Saúde (MS). Rio de janeiro (RJ), Brasil. Mestranda do Programa de Pós-Graduação do Mestrado Profissional em Enfermagem Assistencial da Escola de Enfermagem Aurora Afonso Costa (MPEA/EEAAC) da Universidade Federal Fluminense (UFF). Niterói (RJ), Brasil. E-mail: rnencetti@yahoo.com.br ${ }^{2}$ Enfermeira. Pós-Doutora em Enfermagem. Coordenadora do Programa de MPEA/EEAAC/UFF. Niterói (RJ), Brasil. E-mail: elianeramos.uff@gmail.com ${ }^{3}$ Enfermeira. Professora-Associada da UFF. Vice-Coordenadora do Programa de Pós-Graduação do Mestrado Profissional em Ensino na Saúde MPES/ EEAAC/UFF. Niterói (RJ), Brasil. E-mail: roserosauff@gmail.com

${ }^{4}$ Enfermeira-Paliativista e Chefe da Divisáo de Enfermagem do HC IV/INCA/MS. Rio de janeiro (RJ), Brasil. E-mail: lucianaoliveiraenfer@yahoo.com.br Endereşo para correspondência: Renata Carla Nencetti. Rua Visconde de Santa Isabel, no 274 - Vila Isabel. Rio de janeiro (RJ), Brasil. E-mail: rnencetti@yahoo.com.br
} 


\title{
O Exame Físico na Síndrome de Werdnig-Hoffman: Eficiência nos Cuidados Paliativos
}

The Physical Examination of Werdnig-Hoffman Syndrome: Efficiency in Palliative Care

\author{
El Examen Físico de Werdnig-Hoffman Síndrome: Eficiencia en los Cuidados \\ Paliativos
}

\author{
Monique Aleluia Cayres ${ }^{1}$; Viviane de Melo Souza ${ }^{2}$; Luciana Souza de Castro ${ }^{3}$; Gracieli Braga Moreira Santana ${ }^{4}$; Juliana Pereira Machado ${ }^{5}$ Yasmin de \\ Araújo Pimentel ${ }^{6}$
}

\section{Resumo}

Introduçáo: $\mathrm{O}$ aprimoramento do conhecimento sobre a sistematização da assistência de enfermagem se constitui uma abordagem essencial para a formação do enfermeiro, principalmente quando se depara com pacientes com doenças degenerativas que necessitam da intervenção dos cuidados paliativos para maior conforto para a criança e autonomia para a família auxiliar esse cuidado, melhorando a assistência de enfermagem. Objetivo: Descrever a importância do exame físico no planejamento dos cuidados à criança com Síndrome de Werding-Hoffman. Método: Trata-se de um relato de experiência vivido durante o período acadêmico, no ano de 2016, no setor de emergência pediátrica de um hospital público de uma grande metrópole brasileira. Primeiramente, houve uma reuniâo dos profissionais de saúde para discussão do caso, e, em seguida, foi realizado um exame físico minucioso para o delineamento do plano de cuidados de enfermagem. Resultados: Por se tratar de uma doença sem possibilidades de cura, o exame físico é etapa obrigatória para o planejamento e a execuçáo dos cuidados de enfermagem, pois contribui para identificar alteraçôes biológicas e físicas, pertinentes e importantes nos cuidados paliativos. Conclusáo: Entende-se a necessidade do exame físico para realizar o planejamento da assistência de enfermagem no controle de sintomas, desenvolvendo os cuidados paliativos com eficiência.

Palavras-chave: Atrofias Musculares Espinais da Infância; Exame Físico; Cuidados Paliativos; Cuidados de Enfermagem.

\section{Declaraçáo de Conflito de Interesses: Nada a Declarar.}

\footnotetext{
${ }^{1}$ Enfermeira. Especialista em Enfermagem em Pediatria pela Universidade Federal do Rio de Janeiro (UFRJ). Supervisora Acadêmica do Curso de Enfermagem da Associaçáo Brasileira de Ensino Universitário (Uniabeu). Belford Roxo (RJ), Brasil. E-mail: moniquealeluia@gmail.com

${ }^{2}$ Enfermeira. Especialista em Enfermagem em Neonatologia pela Universidade do Estado do Rio de Janeiro (UERJ). Rio de Janeiro (RJ), Brasil. Supervisora Acadêmica do Curso de Enfermagem da Uniabeu. Belford Roxo (RJ), Brasil. E-mail: enfvivianemelo@gmail.com

${ }^{3}$ Enfermeira. Pós-graduanda em Enfermagem em Pediatria e Neonatologia e Enfermagem Oncológica pela Faculdade Unyleya. Instituto Nacional de Câncer José Alencar Gomes da Silva (INCA)/ Ministério da Saúde (MS). Rio de Janeiro (RJ), Brasil. E-mail: luenf@live.com

${ }^{4}$ Enfermeira. Pós-graduanda em Enfermagem em Pediatria e Neonatologia pela Universidade Estácio de Sá (Unesa). Rio de Janeiro (RJ), Brasil. E-mail: gracieli.braga@hotmail.com

${ }^{5}$ Enfermeira. Pós-graduanda em Enfermagem em Pediatria e Neonatologia pela Faculdade Unyleya. Rio de Janeiro (RJ), Brasil. E-mail: juli_machado30@hotmail.com

${ }^{6}$ Enfermeira. Egressa do Curso de Enfermagem da Uniabeu. Belford Roxo (RJ), Brasil. E-mail: yasminpimentel04@gmail.com

Endereço para correspondência: Luciana Castro. Rua Sargento Antônio Ernesto, no 275, casa 32 - Pavuna. Rio de Janeiro (RJ), Brasil. CEP: $21520-460$.

E-mail: luenf@live.com
} 


\title{
Impacto Econômico dos Opioides no Tratamento da Dor Oncológica em Pacientes em Cuidados Paliativos na Assistência Domiciliar
} Economic Impact of Opioids on the Treatment of Oncological Pain in Patients in Domiciliary Assistance Palliative Care

Impacto Económico de los Opioides en el Tratamiento del Dolor Oncológico en Pacientes de Cuidado Paliativo en Asistencia Domiciliaria

\author{
Rafael Roberto Leira' ; Mariana Ferreira Rodrigues de Souza ${ }^{2}$; Rodrigo Saar da Costa ${ }^{2}$; Maria Fernanda Barbosa ${ }^{2}$
}

\begin{abstract}
Resumo
Introduçáo: A dor crônica configura-se como um dos principais sintomas dos pacientes com câncer avançado, sendo comum a intervenção com opioides fortes. $\mathrm{O}$ acesso a medicamentos e o custo do tratamento figuram como protagonistas na garantia de um cuidado adequado. Objetivo: Descrever o impacto econômico dos opioides fortes no tratamento da dor oncológica em pacientes em cuidados paliativos, na modalidade de assistência domiciliar. Método: Foram analisados os históricos de consumo dos pacientes em cuidados paliativos, na modalidade de assistência domiciliar, no período de maio a novembro de 2015, em um Hospital Federal do Sistema Único de Saúde (SUS). Foi construída uma curva $\mathrm{ABC}$ para avaliação do impacto econômico. A análise estatística foi realizada através de média, mediana e frequência do uso de opioides fortes no período em questão. Resultados: A média de idade foi de 71 anos, sendo 66\% mulheres. Quarenta e dois por cento dos pacientes consumiram opioides fortes e as clínicas de consumo acentuado foram: abdômen, cabeça e pescoço, ginecologia e mastologia. O impacto econômico dos opioides fortes foi de $26 \%$ do custo total com medicamentos no período analisado. Conclusáo: No contexto dos cuidados paliativos, o uso de opioides fortes representa importante estratégia terapêutica no manejo da dor e relevante impacto econômico. Assim, observa-se a necessidade da utilização desses medicamentos em consonância com protocolos e diretrizes clínicas e apropriadas estratégias de financiamento permitindo a expansão da oferta de opioides fortes em todos os serviços de assistência domiciliar.

Palavras-chave: Cuidados Paliativos; Neoplasias/terapia; Analgésicos Opióides/uso terapêutico; Assistência Domiciliar; Farmacoeconomia.
\end{abstract}

\section{Declaraçáo de Conflito de Interesses: Nada a Declarar.}

\footnotetext{
${ }^{1}$ Residente do Programa de Residência Multiprofissional em Oncologia do Instituto Nacional de Câncer José Alencar Gomes da Silva (INCA)/Ministério da Saúde (MS). Rio de Janeiro (RJ), Brasil.

${ }^{2}$ Farmacêuticos do Hospital do Câncer IV (HC IV)/INCA/MS. Rio de Janeiro (RJ), Brasil.

Endereço para correspondência: Maria Fernanda Barbosa. Rua Visconde de Santa Isabel, no 274. Vila Isabel. Rio de Janeiro (RJ), Brasil. CEP: $20560-120$.

E-mail: mbarbosa@inca.gov.br.
} 


\section{Perfil do Paciente em Cuidado Paliativo Oncológico Assistido pela Fisioterapia na Internação Hospitalar do Instituto Nacional de Câncer José Alencar Gomes da Silva IV Profile of the Patient in Oncology Palliative Care Assisted by Physiotherapy in the Hospital Admission of the National Cancer Institute JoséAlencar Gomes da Silva IV Perfil del paciente en Cuidado Paliativo Oncológico Asistido por la Fisioterapia en Internación Hospitalar del Instituto Nacional de Cáncer José Alencar Gomes da Silva IV}

Francine Peres da Silva'; Patricia Almeida Chelles ${ }^{2}$

\section{Resumo}

Introdução: A assistência da fisioterapia na internação auxilia no controle de sintomas, minimiza complicaçóes da doença ou do tratamento e contribui para a qualidade de vida dos pacientes. Objetivo: Compreender o perfil dos pacientes para assistência fisioterapêutica na internação hospitalar em cuidados paliativos oncológicos. Método: Estudo descritivo, realizado no período de 1/12/2015 a 5/3/2016. Coleta de dados em prontuários na enfermaria de cuidado paliativo do Instituto Nacional do Câncer José Alencar Gomes da Silva (INCA). Variáveis utilizadas para construção do perfil: clínica de origem; escala de Performance Status de Karnofsky (KPS); motivo da internação pertinente à atuação da fisioterapia: dor (neuropática, miofascial, óssea e articular), dispneia não terminal, hipersecreção traqueobrônquica, metástase óssea, síndrome de compressão medular, fratura patológica, trombose venosa profunda, linfedema, entre outras (avaliação funcional, risco de queda) e a necessidade de acompanhamento pela fisioterapia. Resultados: Avaliados 589 prontuários. Desses, 144 pacientes $(24,4 \%)$ tinham motivo para assistência fisioterapêutica. A clínica de maior prevalência foi a de ginecologia representando $25 \%$ dos atendimentos realizados. O KPS variou entre 30\% e 50\%. Os motivos de internaçáo mais prevalentes foram: dor (24,30\%) e dispneia (22,91\%). Apenas 22,22\% dos pacientes apresentaram a necessidade de continuidade da assistência durante a internaçáo. Conclusáo: $\mathrm{O}$ estudo permitiu obter o perfil do paciente em cuidado paliativo oncológico na internaçáo e selecionar a demanda para Fisioterapia. O conhecimento do perfil do paciente favorece o planejamento da assistência, tornando-a individualizada e voltada para prioridades. Acrescem-se, ainda, o favorecimento de cumprimento de metas, de utilização adequada de recursos humanos e financeiros e o redirecionamento de indicadores.

Palavras-chave: Cuidados Paliativos; Doente Terminal; Neoplasias/terapia; Serviço Hospitalar de Fisioterapia; Institutos de Câncer.

\footnotetext{
${ }^{1}$ Fisioterapeuta. Pós-Graduada em Fisioterapia Neurofuncional pela Universidade Estácio de Sá (Unesa). Hospital do câncer IV (HC IV)/ Unidade de Cuidado Paliativo do Instituto Nacional de Câncer José Alencar Gomes da Silva (INCA)/Ministério da Saúde (MS). Rio de Janeiro (RJ), Brasil. E-mail: franzperes74@gmail.com

${ }^{2}$ Fisioterapeuta. Pós-Graduada em Fisioterapia Pneumofuncional pela Universidade Gama Filho (UGF). Unidade de Cuidado Paliativo do HC IV/ INCA/MS. Rio de Janeiro (RJ), Brasil.E-mail: patricia.chelles@inca.gov.br

Enderę̧o para correspondência: Patricia Almeida Chelles. Rua Visconde de Santa Isabel, no 274, Vila Isabel. Rio de Janeiro (RJ), Brasil. CEP: $20560-120$.

E-mail: patricia.chelles@inca.gov.br
} 